\title{
Long-term survival of the middle and lower thoracic esophageal cancer patients after surgical treatment through left or right thoracic approach
}

\author{
Ding Yang, You-Sheng Mao, Jie He, Shu-Geng Gao, Ke-Lin Sun, Ju-Wei Mu, Qi Xue, Da-Li Wang, Yu- \\ Shun Gao, Jun Zhao, Xiang-Yang Liu, De-Kang Fang, Jian Li, Yong-Gang Wang, Liang-Ze Zhang, Jin- \\ Feng Huang, Bing Wang \\ Department of Thoracic Surgery, National Cancer Center/Cancer Hospital, Chinese Academy of Medical Sciences and Peking Union Medical \\ College, Beijing 100021, China \\ Contributions: (I) Conception and design: D Yang, YS Mao; (II) Administrative support: All authors; (III) Provision of study materials or patients: All \\ authors; (IV) Collection and assembly of data: D Yang, YS Mao; (V) Data analysis and interpretation: D Yang, YS Mao; (VI) Manuscript writing: All \\ authors; (VII) Final approval of manuscript: All authors. \\ Correspondence to: You-Sheng Mao. Department of Thoracic Surgery, National Cancer Center/Cancer Hospital, Chinese Academy of Medical \\ Sciences and Peking Union Medical College, Beijing 100021, China. Email: maoysherx@qq.com.
}

Background: Esophageal cancer is one of the most prevalent malignancies with a high incidence and mortality in China, the main treatment for esophageal cancer at present is still surgery-based multimodality treatment, and surgery is still the most effective measure. However, the modes of surgical treatment for esophageal cancer have been diverse. The surgical approaches can be mainly divided into the left thoracic approach and right thoracic approach in China. The long-term survival of the patients treated through right approach was reported better than that through left thoracic approach, but until now no statistically significant difference was found between two approaches, especially, for those with middle and lower thoracic esophageal cancer without suspected upper mediastinal lymph node metastasis in preoperative examinations, no definite conclusion have been made on selection of the approach, therefore, this studies try to compare the long-term survival between two approaches .

Methods: The data of 402 cases with complete resection and two-field lymph node dissection from January, 2011 to December, 2011 in the Cancer Hospital, Chinese Academy of Medical Sciences was retrospectively reviewed and analyzed. Propensity score matching (PSM) analysis and life-table in SPSS 22.0 and Stata 14.0 were used to analyze the survival.

Results: Totally, 402 cases were surgically treated either via left or right thoracic approach. The overall 5 -year survival rate of this series was $38 \%$, it was $37 \%$ in 281 cases surgically treated through left approach, and $39 \%$ in 121 cases through right approach $(\mathrm{P}=0.908)$. The 5 -year survival of 256 patients without suspected lymph node metastasis in the upper mediastinum based on the preoperative examinations surgically treated through left approach was $38 \%$ versus $43 \%$ of 88 cases through right approach $(\mathrm{P}=0.404)$. After PSM, the 5-year survival of 110 cases surgically treated through left approach was $32 \%$ versus $40 \%$ of another matched 110 cases through right approach $(\mathrm{P}=0.146)$. for the patients without suspected lymph node metastasis in the upper mediastinum based on preoperative examinations, the 5-year survival of 88 surgically treated through left approach was $33 \%$ versus $44 \%$ of another matched 88 cases through right approach $(\mathrm{P}=0.239)$.

Conclusions: For the middle and lower thoracic esophageal cancer patients, whether or not who has suspected lymph node metastasis in the upper mediastinum based on preoperative CT and EUS, the surgical treatment through right thoracic approach can achieve better but not significantly better overall survival than that through left thoracic approach. Further prospective randomized clinical trials are still needed to verify this disputed issue on approach selection. 
Keywords: Esophageal cancer; survival; surgical treatment; prognosis; propensity score matching (PSM)

Submitted Oct 03, 2017. Accepted for publication Apr 01, 2018.

doi: $10.21037 /$ jtd.2018.04.45

View this article at: http://dx.doi.org/10.21037/jtd.2018.04.45

\section{Introduction}

Esophageal cancer is one of the most prevalent cancers in China. The incidence of esophageal cancer in 2012 was 21.17/100 thousand in China reported in 2015 by Chen et al. which ranked the fifth position, and the mortality rate was $15.58 / 100$ thousand, ranked at the fourth position among all malignant tumors in China $(1,2)$. Currently, the major treatment mode for esophageal carcinoma is still the surgery-based multimodality treatment, and surgery is still one of the most effective treatment measures at present. However, the operation modes for esophageal carcinoma have been complex and diverse in China. The most applied surgical approach for esophageal cancer was left thoracic approach and the right thoracic approach was rarely used only for the patients with upper thoracic esophageal cancer during the past several decades (3).

It was reported that the extent of lymph node dissection significantly affects the long-term survival of esophageal cancer patients and the number of lymph node metastasis and the lesion location were independent prognostic factors (4-7). Therefore, standardized complete lymph node dissection is critical for the prognosis of esophageal carcinoma. And the key to complete lymph node dissection lies in the choice of surgical approach. It is much easier to remove all chest lymph nodes along the esophageal drainage area through the right thoracic approach than through the left thoracic approach due to anatomic difference in the two chest cavities, therefore, theoretically, the patients surgically treated through right chest should have better long-term survival than that through left thoracic chest. However, several retrospective studies reported that no statistically significant difference existed between the two approaches in the long-term survival, and the left transthoracic approach is even superior in some aspects in the treatment of lymph node negative ESCC (8,9). Compared with the left thoracic approach, right thoracic approach do has some advantages in clearing the chest lymph nodes, however, it has some disadvantage such as more complicated operation procedure and longer operation time, more bleeding, higher incidence of postoperative complications and slower recovery, etc., especially when the lymph nodes along both recurrent laryngeal nerve are dissected completely, it frequently results in palsy of recurrent laryngeal nerves, hoarseness and week coughing, which affect the postoperative rehabilitation of the patient severely. Therefore, for the patients with middle and lower thoracic esophageal carcinoma without suspected lymph nodes metastasis in the upper mediastinum by preoperative examinations, which approach is more beneficial, more evidences are still needed to prove it.

\section{Methods}

A total of 402 surgically treated patients with middle and lower thoracic esophageal carcinoma in Cancer Hospital, Chinese Academy of Medical Sciences from January 1, 2011 to December 31, 2011 were enrolled into this retrospective study on the following criterion: squamous cell carcinoma of the middle and lower thoracic esophagus confirmed by postoperative pathology; radical resection with chest and abdominal 2-field lymph node dissection; no preoperative adjuvant therapy; no history of other malignancies; no distant metastasis detected before operation. Of the 402 cases, 59 were assessed as having suspected superior mediastinal lymph node metastasis by preoperative enhanced high resolution and thin CT and EUS; and 344 as negative. All patients' lesions were resectable and staged by TNM staging system of AJCC eighth edition by preoperative examinations. All patients underwent a radical resection of esophageal carcinoma with thoracic and abdominal 2-field lymph node dissection, and had esophagogastric anastomosis in the chest or neck. The patients with only exploratory or palliative resection were excluded. Of these 402 cases, 281 were treated by left thoracotomy, including left posterolateral incision in 273 cases and left posterolateral thoracotomy + left neck incision in 9 cases, and the other 121 by right thoracic approach, including Ivor-Lewis in 18 cases, McKeown in 80 cases and thoracoscopic/laparoscopic minimally invasive resection in 23 cases.

The postoperative survival status was confirmed by outpatient review records and telephone follow-up and census registration. The longest observation period was 
Table 1 Characteristics of the patients treated by left and right thoracic approach

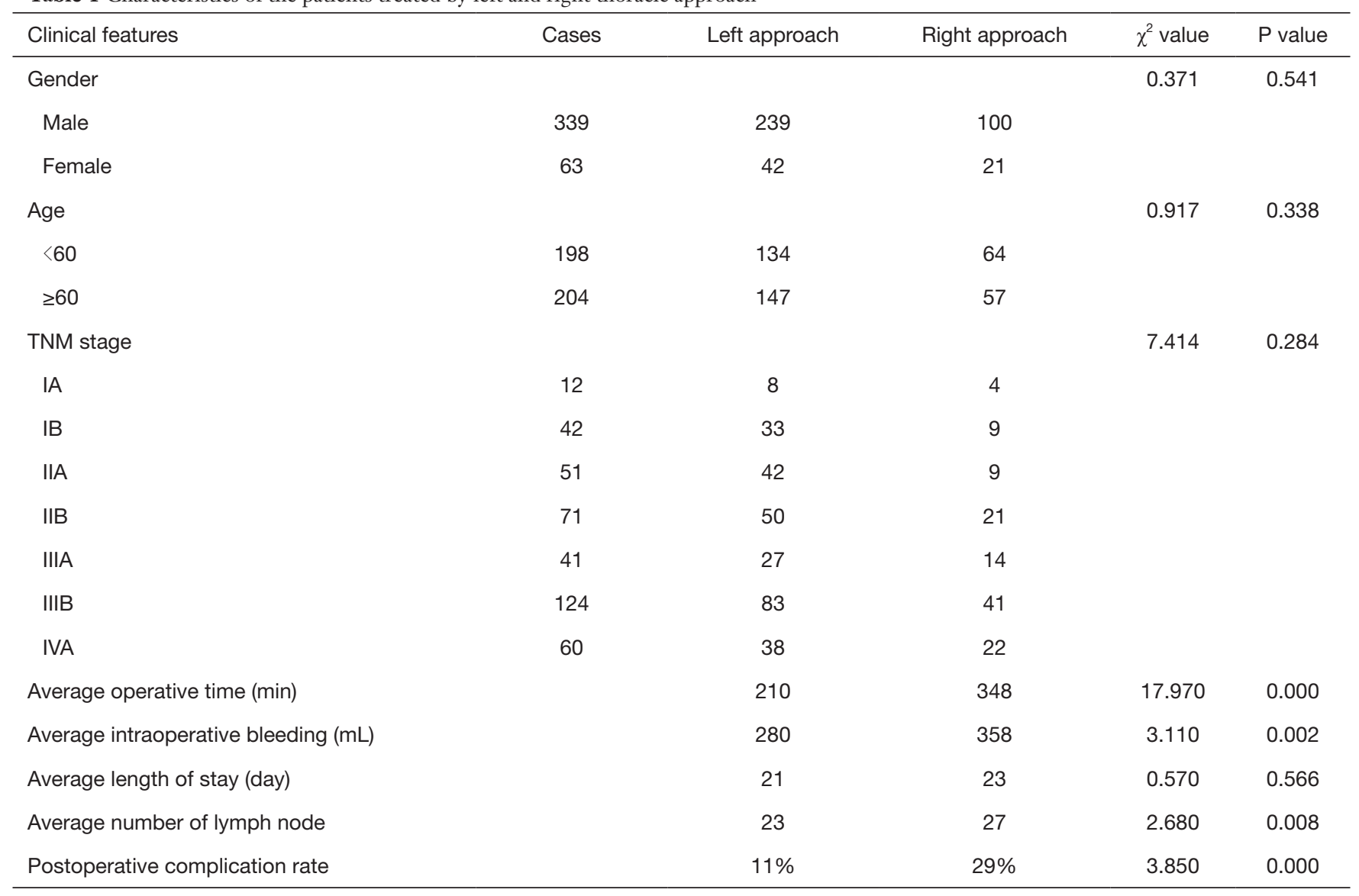

60 months. The final follow-up was completed on April 1, 2017 , and the follow-up rate was $93 \%$.

The survival of the patients treated by different approaches was analyzed by SPSS 22, and survival was calculated by the life-table method, the survival among the groups was compared by Wilcoxon method, $\mathrm{P}<0.05$ was considered as statistically significant. In order to eliminate bias between the left and right approach groups, propensity score matching (PSM) was used to match two approach groups and the survival analysis was compared again after PSM. The Stata 14 software was used to assess propensity score on gender, age, and preoperative $T$, and $\mathrm{N}$ stage, postoperative complications, and 1:1 pairing was made based on similar propensity values. The caliper value was set to 0.05 , matching according to propensity score, respectively, the patients by left/right thoracic approach were paired first, and then the patients without upper mediastinal lymph node metastasis were paired.

This retrospective study was approved by Ethics Committee of National Cancer Center/Cancer Hospital
(NCC201802006).

\section{Results}

In total, 281 patients treated by left thoracic approach including 239 males and 42 females; 121 patients treated by right thoracic approach consisting of 100 males and 21 females. The patients' characteristics of two approaches were summarized in the Table 1 , the average operative time, intraoperative bleeding, length of hospital stay, number of resected lymph node and postoperative complication rate in the left thoracic approach group was $210 \mathrm{~min}$, $280 \mathrm{~mL}, 21$ days, 23, 11\% versus $348 \mathrm{~min}, 358 \mathrm{~mL}$, 23 days, 27 , and $29 \%$ in the right thoracic approach group, respectively. There was statistically significant difference in average operative time $(\mathrm{P}<0.0001)$, average intraoperative bleeding $(\mathrm{P}=0.002)$, average number of resected lymph node $(\mathrm{P}<0.008)$ and postoperative complications $(\mathrm{P}<0.0001)$. Twenty-four of 33 cases in the right approach group with suspicious metastasis of right superior mediastinal lymph 


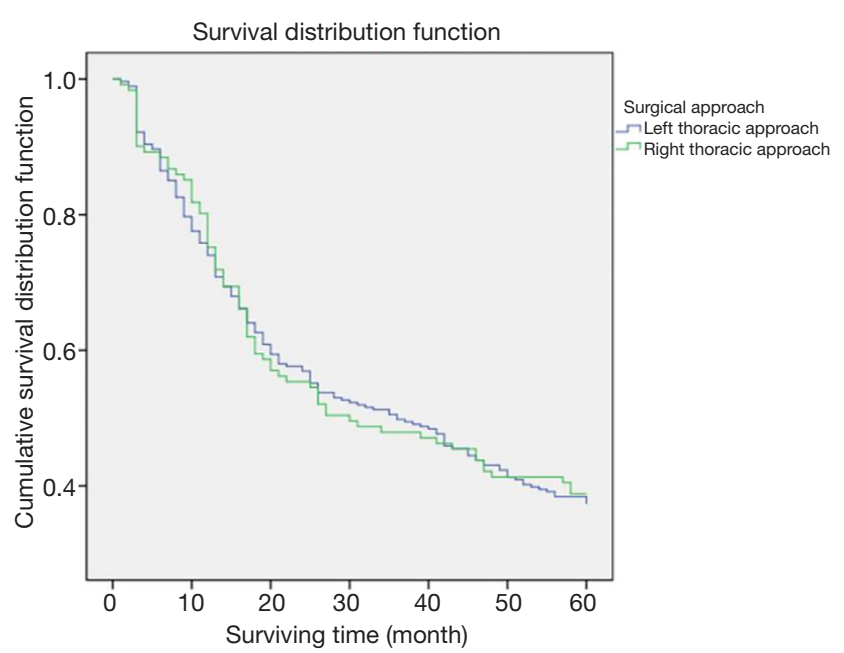

Figure 1 Survival curves of different approach groups.

Table 2 Survival analysis result of different approach groups

\begin{tabular}{|c|c|c|c|c|}
\hline \multirow[b]{2}{*}{ Variables } & \multicolumn{2}{|c|}{ Surgical approach } & \multirow[b]{2}{*}{ df } & \multirow[b]{2}{*}{$P$ value } \\
\hline & $\begin{array}{l}\text { Left thoracic } \\
\text { approach }\end{array}$ & $\begin{array}{c}\text { Right thoracic } \\
\text { approach }\end{array}$ & & \\
\hline $\begin{array}{l}\text { 5-year survival } \\
\text { rate }(\%)\end{array}$ & 37 & 39 & 1 & 0.908 \\
\hline Overall survival & & 38 & & \\
\hline
\end{tabular}

nodes by preoperative examinations, were confirmed to have metastasis by postoperative pathology, with an accuracy rate of $72 \%$. The postoperative death rate within 30 days was $1.11 \%$ in the left approach group and $1.17 \%$ in the right approach group without statistically significant difference between two groups $(\mathrm{P}=0.334)$.

The 5-year survival of 281 patients surgically treated by left thoracic approach was $37 \%$, versus $39 \%$ in 121 patients by right thoracic approach ( $\mathrm{P}>0.05$, Figure 1, Table 2).

In the patients who had no suspected metastatic superior mediastinal lymph nodes based on preoperative CT, the 5 -year survival of 256 surgically treated by left thoracic approach was $38 \%$ versus $43 \%$ of 88 patients by right thoracic approach $(\mathrm{P}>0.05$, Figure 2, Table 3$)$.

In order to eliminate the bias between the left and right approach group, the patients in in these two groups underwent 1:1 matching based on age, gender, preoperative $\mathrm{T}$ staging, preoperative $\mathrm{N}$ staging, postoperative complications by PSM, eventually 110 cases in each thoracic

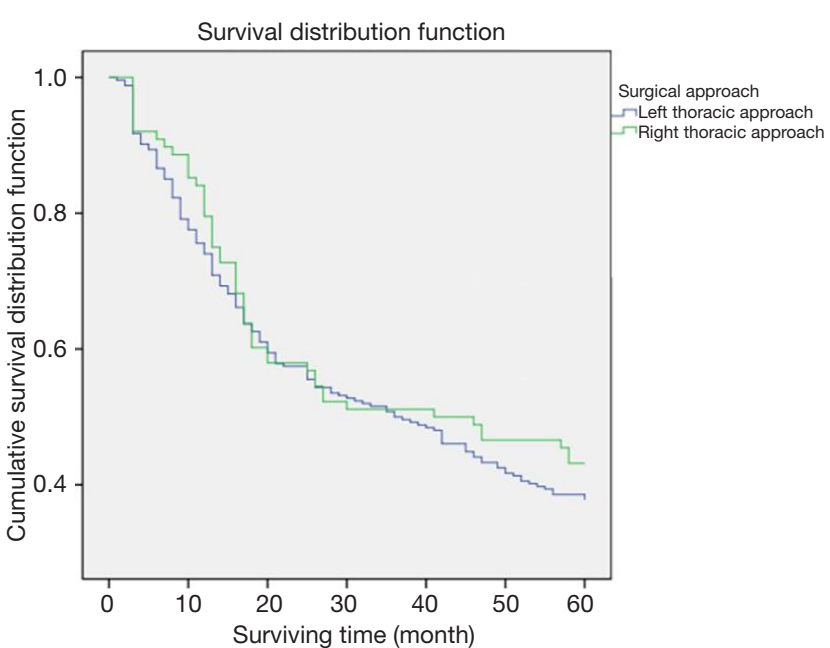

Figure 2 Survival curves of different approach groups with no suspected metastatic superior mediastinal lymph nodes.

Table 3 Survival analysis result of different approach groups with no suspected metastatic superior mediastinal lymph nodes

\begin{tabular}{|c|c|c|c|c|}
\hline \multirow[b]{2}{*}{ Variables } & \multicolumn{2}{|c|}{ Surgical approach } & \multirow[b]{2}{*}{$d f$} & \multirow[b]{2}{*}{$P$ value } \\
\hline & $\begin{array}{c}\text { Left thoracic } \\
\text { approach }\end{array}$ & $\begin{array}{l}\text { Right thoracic } \\
\text { approach }\end{array}$ & & \\
\hline $\begin{array}{l}5 \text {-year survival } \\
\text { rate }(\%)\end{array}$ & 38 & 43 & 1 & 0.404 \\
\hline Overall survival & \multicolumn{2}{|c|}{40} & & \\
\hline
\end{tabular}

approach group were matched, no statistically significant difference in the characteristics was revealed between two groups after matching (Table 4). The 5-year survival rate of the left thoracic approach group was $32 \%$ versus $40 \%$ in the right thoracic approach group $(\mathrm{P}>0.05)$, there was still no statistically significant difference between the two groups (Figure 3, Table 5).

Eighty-eight patients who had no suspected metastatic superior mediastinal lymph nodes by preoperative examinations in each approach group were finally matched (Table 6), the 5 -year survival rate of the left thoracic approach group was $33 \%$ versus $44 \%$ in the right thoracic approach group $(\mathrm{P}>0.05)$, there was still no statistically significant difference between the two groups (Figure 4, Table 7).

\section{Discussion}

Esophageal carcinoma is one of the most prevalent 
Table 4 PSM statistics of different approach groups

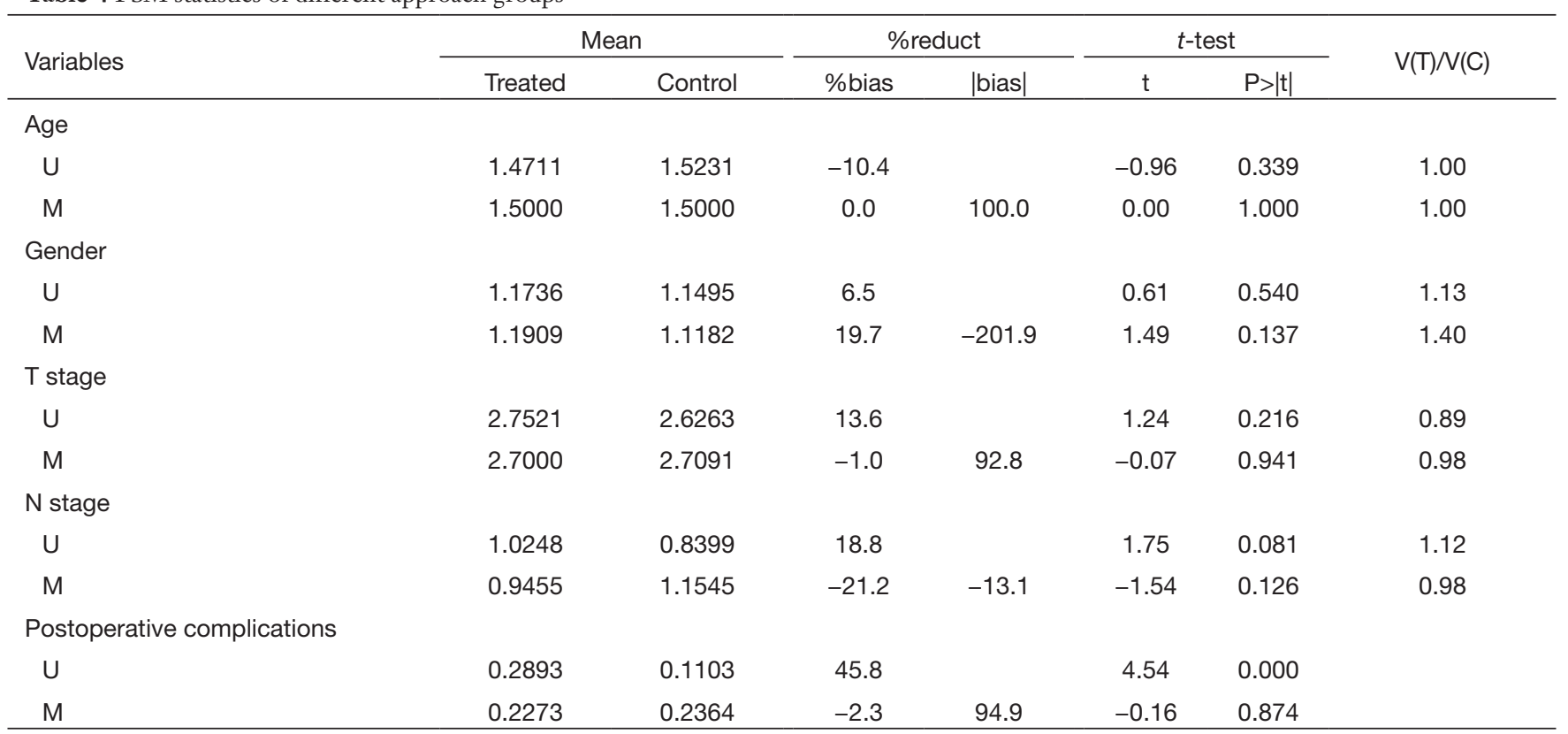

PSM, propensity score matching; $U$, unmatched; $M$, matched.

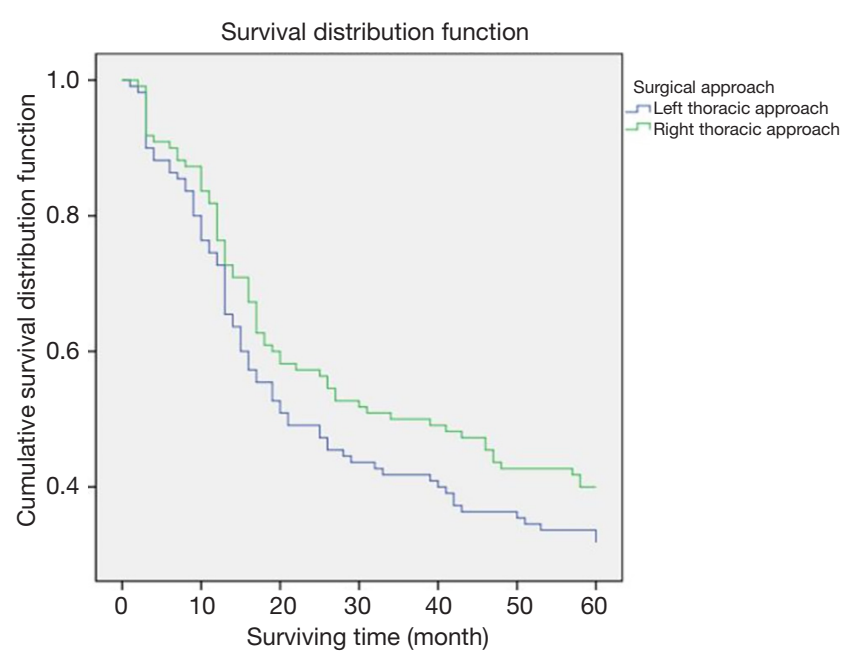

Figure 3 Survival curves of left and right thoracic approach groups after PSM. PSM, propensity score matching

malignant tumors in China. Currently the major treatment mode for esophageal carcinoma is still the surgery-based multimodality treatment (10). For the patients with a resectable lesion, if treated by surgery, complete resection of the lesion in the esophagus and all drainage lymphatic tissue are the basic requirements (11). The 5 -year survival rate reported in the literatures was better through right thoracic approach than that through left thoracic approach (12-15),
Table 5 Survival analysis result of different approach groups after PSM

\begin{tabular}{|c|c|c|c|c|}
\hline \multirow[b]{2}{*}{ Variables } & \multicolumn{2}{|c|}{ Surgical approach } & \multirow[b]{2}{*}{$d f$} & \multirow[b]{2}{*}{$P$ value } \\
\hline & $\begin{array}{c}\text { Left thoracic } \\
\text { approach }\end{array}$ & $\begin{array}{l}\text { Right thoracic } \\
\text { approach }\end{array}$ & & \\
\hline $\begin{array}{l}5 \text {-year survival } \\
\text { rate }(\%)\end{array}$ & 32 & 40 & 1 & 0.146 \\
\hline Overall survival & \multicolumn{2}{|c|}{36} & & \\
\hline
\end{tabular}

PSM, propensity score matching.

the possible reason might be that through right thoracic approach the lymph nodes in the upper mediastinum, especially the lymph nodes along the recurrent laryngeal nerves can be easily and completely dissected, which may reduce the possibility to develop recurrence in some patients. However, there was no statistically significant difference between two approaches in most retrospective studies, especially in those without suspected metastatic lymph nodes in the upper mediastinum, which is similar to the results of this series, $37 \%$ through left thoracic approach versus $39 \%$ through the right thoracic approach $(\mathrm{P}>0.05)$.

Why there was no statistically significant difference between the right and left thoracic approach in the most of retrospective studies, the possible explanation might 
Table 6 PSM statistics of different approach groups with no suspected metastatic superior mediastinal lymph nodes

\begin{tabular}{|c|c|c|c|c|c|c|c|}
\hline Variables & \multicolumn{2}{|c|}{ Mean } & \multicolumn{2}{|c|}{ \%reduct } & \multicolumn{2}{|c|}{$t$-test } & $\mathrm{V}(\mathrm{T}) / \mathrm{V}(\mathrm{C})$ \\
\hline \multicolumn{8}{|l|}{ Age } \\
\hline$U$ & 1.4545 & 1.5156 & -12.2 & & -0.99 & 0.324 & 1.00 \\
\hline M & 1.4598 & 1.4943 & -6.9 & 43.5 & -0.45 & 0.651 & 0.99 \\
\hline$U$ & 1.1705 & 1.1602 & 2.8 & & 0.23 & 0.822 & 1.06 \\
\hline M & 1.1724 & 1.1609 & 3.1 & -11.6 & 0.20 & 0.840 & 1.06 \\
\hline \multicolumn{8}{|l|}{ T stage } \\
\hline $\mathrm{U}$ & 2.6818 & 2.6016 & 8.4 & & 0.68 & 0.495 & 1.01 \\
\hline$U$ & 1.0114 & 0.8047 & 20.7 & & 1.72 & 0.086 & 1.22 \\
\hline M & 0.9885 & 1.0805 & -9.2 & 55.5 & -0.58 & 0.564 & 0.92 \\
\hline \multicolumn{8}{|c|}{ Postoperative complications } \\
\hline$U$ & 0.2727 & 0.1094 & 42.3 & & 3.75 & 0.000 & \\
\hline M & 0.2644 & 0.2644 & 0.0 & 100.0 & 0.00 & 1.000 & \\
\hline
\end{tabular}

PSM, propensity score matching; U, unmatched; M, matched.

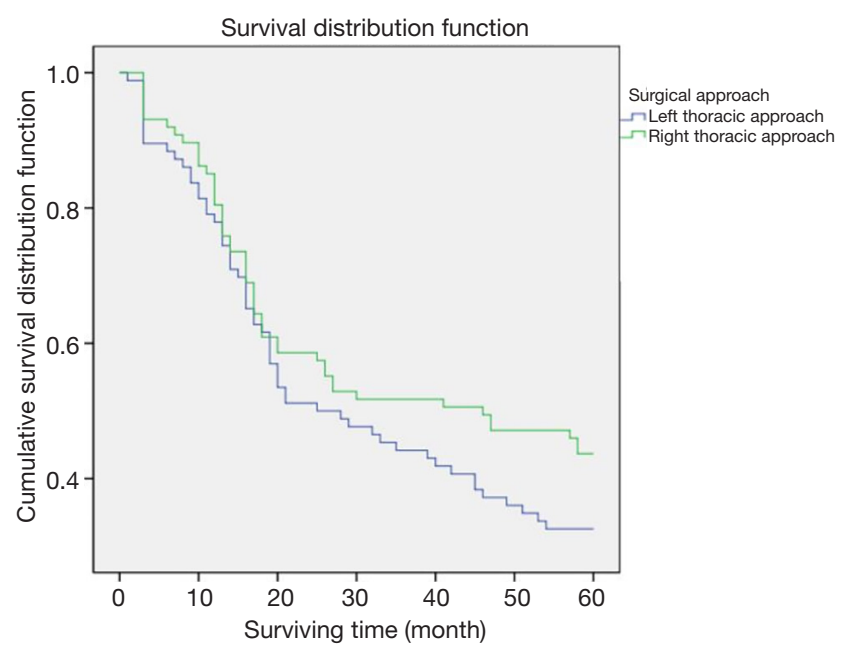

Figure 4 Survival curves of different approach groups with no suspected metastatic superior mediastinal lymph nodes after PSM. PSM, propensity score matching.
Table 7 Survival analysis result of two different approach groups with no suspected metastatic superior mediastinal lymph nodes after PSM

\begin{tabular}{|c|c|c|c|c|}
\hline \multirow[b]{2}{*}{ Variables } & \multicolumn{2}{|c|}{ Surgical approach } & \multirow[b]{2}{*}{ df } & \multirow[b]{2}{*}{$P$ value } \\
\hline & $\begin{array}{c}\text { Left thoracic } \\
\text { approach }\end{array}$ & $\begin{array}{l}\text { Right thoracic } \\
\text { approach }\end{array}$ & & \\
\hline $\begin{array}{l}5 \text {-year survival } \\
\text { rate }(\%)\end{array}$ & 33 & 44 & 1 & 0.239 \\
\hline Overall survival & \multicolumn{2}{|r|}{38} & & \\
\hline
\end{tabular}

PSM, propensity score matching.

be that the patients underwent esophagectomies through right thoracic approach usually have more advanced preoperative $\mathrm{N}$ stage, especially the patients may have enlarged lymph nodes in the upper mediastinum in previous retrospective studies, and the other reasons might be that longer operative time, more intraoperative blood loss and more postoperative complications through right thoracic approach partially offset the survival benefit brought by more complete lymph node dissection through right thoracic approach. Therefore, in order to decrease the 
adverse effect of the above factors on long-term survival in the right thoracic approach group, PSM analysis was used, and the patients in the two approach groups were matched 1:1 according to the propensity scores based on basic clinical characteristics such as age, gender, preoperative $\mathrm{T}$ stage, and $\mathrm{N}$ stage and postoperative complications. The 5 -year survival rate of the patients treated by the left thoracic approach was $32 \%$ versus $40 \%$ by the right thoracic approach after PSM $(\mathrm{P}=0.146)$. Though the 5-year survival rate of the right thoracic approach group was much better than that of left thoracic approach group, there was still no statistically significant difference between two groups. In addition to the above factors, when suspicious lymph node metastasis in the upper mediastinum was added for PSM, the 5 -year survival rate of the left thoracic approach group was $33 \%$ versus $44 \%$ of the right thoracic approach group, there was still no statistically significant difference between two approaches, which is similar to the results, $46.56 \%$ versus $48.35 \%(\mathrm{P}=0.388)$, reported by $\mathrm{Ma}$ et al. (9). The authors suggested that the left thoracic approach is still an alternative options for the patients who has no suspected lymph nodes in the upper mediastinum due to similar long-term survival and local recurrence patterns either by the right or the left thoracic approach. However, in the reported series, more than $60 \%$ patients had stage I-II lesions who may have lower rate of lymph node metastasis in the upper mediastinum, while in this series, the majority of the patients had more advanced stage lesions, therefore, the results are not comparable to the results reported in the literatures and the prospective controlled randomized clinical trials are still required to prove the preservation of left thoracic approach for the patients who has no suspected lymph nodes in the upper mediastinum.

Currently, radical resection of esophageal carcinoma and complete thoracic lymph nodes dissection through right thoracic approach has been gradually accepted and become more and more popular due to extensive application of VATS esophagectomies in China. less intraoperative bleeding, similar operation time and shorter hospital stay of VATS esophagectomies through right approach has overcome the disadvantages of the routine right thoracotomy in the past decades, with more complete lymph nodes dissection and less perioperative complications, therefore, the advantage of right thoracic approach will be more significant. However, further prospective randomized controlled studies are still required to verify whether or not the right thoracic approach has a statistically significant advantage in improving long-term survival.
In conclusion, for the patients with middle and lower thoracic esophageal cancer, whether or not who has suspected lymph node metastasis in the upper mediastinum based on preoperative CT and EUS, the surgical treatment through right thoracic approach can achieve better but not significantly better overall survival than that through left thoracic approach. Further prospective randomized clinical trials are still needed to verify this disputed issue on approach selection.

\section{Acknowledgements}

Funding: This work is supported by grants from National Science and Technology Support Program (2015BAI12B08, YS Mao).

\section{Footnote}

Conflicts of Interest: The authors have no conflicts of interest to declare.

Ethical Statement: This retrospective study was approved by Ethics Committee of National Cancer Center/Cancer Hospital (NCC201802006).

\section{References}

1. Chen W, Zheng R, Zhang S, et al. Report of Cancer Incidence and Mortality in China, 2012. Chin J Cancer 2016;35:73.

2. Chen $W$, Zheng R, Baade PD, et al. Cancer statistics in China, 2015. CA Cancer J Clin 2016;66:115-32.

3. He J. Thoracic oncology. Beijing: People's Medical Publishing House, 2013.

4. Hagen JA, DeMeester SR, Peters JH, et al. Curative resection for esophageal adenocarcinoma: analysis of 100 en bloc esophagectomies. Ann Surg 2001;234:520-30; discussion 530-1.

5. Shimada H, Okazumi S, Matsubara H, et al. Impact of the number and extent of positive lymph nodes in 200 patients with thoracic esophageal squamous cell carcinoma after three-field lymph node dissection. World J Surg 2006;30:1441-9.

6. Chen Y, Chen J, Zhu K, et al. The relationship between number of metastatic lymph node and prognosis of thoracic-esophageal cancer patients treated with radical resection. Chin J Thorac Cardiovasc Surg 2014;30:76-8.

7. Xiao ZF, Yang ZY, Wang LH, et al. Influence of the 
number of lymph node metastasis on survival and significance of postoperative radiotherapy for esophageal carcinoma. Zhonghua Zhong Liu Za Zhi 2004;26:112-5.

8. Ma Q, Liu W, Long H, et al. Right versus left transthoracic approach for lymph node-negative esophageal squamous cell carcinoma. J Cardiothorac Surg 2015;10:123.

9. Ma J, Zhan C, Wang L, et al. The sweet approach is still worthwhile in modern esophagectomy. Ann Thorac Surg 2014;97:1728-33.

10. He J. Clinical practice guidelines for the diagnosis and treatment of esophageal cancer. Beijing: Peking Union Medical College Press, 2013.

11. Mao Y, He J, Gao S, et al. Controversies in the surgical treatment for esophageal carcinoma and future investigation. Zhonghua Wei Chang Wai Ke Za Zhi 2015;18:851-4.

12. Mao YS, He J, Dong JS, et al. Comparison of the results

Cite this article as: Yang D, Mao YS, He J, Gao SG, Sun KL, Mu JW, Xue Q, Wang DL, Gao YS, Zhao J, Liu XY, Fang DK, Li J, Wang YG, Zhang LZ, Huang JF, Wang B. Long-term survival of the middle and lower thoracic esophageal cancer patients after surgical treatment through left or right thoracic approach. J Thorac Dis 2018;10(5):2648-2655. doi: 10.21037/ jtd.2018.04.45 of lymph node dissection via left versus right thoracotomy. Zhonghua Zhong Liu Za Zhi 2012;34:296-300.

13. Luo KJ, Fu JH, Hu Y, et al. Efficacy of surgical resection of left and right transthoracic approaches for middle thoracic esophageal squamous cell carcinoma. Ai Zheng. 2009;28:1260-4.

14. Li B, Xiang J, Zhang Y, et al. Comparison of IvorLewis vs Sweet esophagectomy for esophageal squamous cell carcinoma: a randomized clinical trial. JAMA Surg 2015;150:292-8.

15. Li B, Hu H, Zhang Y, et al. Extended Right Thoracic Approach Compared With Limited Left Thoracic Approach for Patients With Middle and Lower Esophageal Squamous Cell Carcinoma: Three-year Survival of a Prospective, Randomized, Open-label Trial. Ann Surg 2018;267:826-32. 